\title{
Avaliação Radiográfica e Histomorfométrica da Mandíbula de Ratas Medicadas com Glicocorticóide e Bifosfonato
}

Mandible's Radiographic and Histomorphometric Assessment in Female Rats Medicated with Glucocorticoid and Biphosphonate

\author{
Celia Regina Winck Mahl ${ }^{1}$, Fernanda Goulart da Fontoura ${ }^{2}$, Priscilla Vendramini Borelli ${ }^{3}$, Isabel Lauxen da Silva ${ }^{4}$, Vania Fontanella ${ }^{5}$
}

\begin{abstract}
Glucocorticoids and biphosphonates are drugs that interfere in bone structure. In order to assess if these changes are evident at radiographies and histological slides, 36 wistar female rats were allocated to 3 groups of 12 animals. Group 1 received saline solution (subcutaneous injections - $2 \mathrm{ml} / \mathrm{kg}$ ), groups 2 and 3 received metilprednisolone acetate $(1 \mathrm{mg} / \mathrm{kg})$. Group 3 received also risedronate $(3 \mathrm{mg} / \mathrm{kg})$. The animals were killed, the left hemimandíble was dissected, radiographed and histologically prepared. One observer blind to the group composition measured the optical density and trabecular proportion means. The ANOVA, complemented by Tukey's test $(\alpha=5 \%)$ showed significantly smaller mean in the group 2 (glucocorticoid, $165.54 \pm 8.95$ ) than in groups 1 (control, 182.96 \pm 4.53 ) and $3(180.99 \pm 6.21)$. The groups 1 and 3 do not differ between themselves. The same statistical tests showed significant histological differences among groups. Group 2 (glucocorticoid, $53.94 \pm 12.53$ ) had the smallest mean proportion of bone trabeculae per field, and group 3 (risedronate, 81.14 \pm 7.20 ) the largest. Both differ significantly from group 1 (control, 72.00 \pm 5.95 ) $(F=37.56 ; p<0.01)$. In conclusion, glucocorticoid and biphosphonate administration interferes with rats mandibular trabecular proportion and these changes are radiographically detectable..
\end{abstract}

Keywords: Osteoporosis; Rat; Mandible; Radiography.

\section{Resumo}

Glicocorticóides e bifosfonatos são medicamentos que interferem na estrutura óssea. Para avaliar se estas mudanças se manifestam radiográfica e histologicamente na mandíbula, foram utilizadas 36 ratas Wistar, divididas em três grupos de 12 animais. O grupo 1 (controle) recebeu injeções subcutâneas $(2 \mathrm{ml} / \mathrm{kg})$ de solução salina, os grupos 2 e 3 receberam acetato de metilprednisolona (1 $\mathrm{mg} / \mathrm{kg}$ ), sendo que o grupo 3 recebeu adicionalmente risedronato $(3 \mathrm{mg} / \mathrm{kg})$. Os animais foram mortos, a hemi-mandíbula esquerda foi dissecada, radiografada e submetida a processamento histológico. Um observador, cego para o grupo a que pertencia a imagem, obteve a média de densidade óptica e a proporção de trabéculas ósseas por campo. A ANOVA, complementada pelo Teste de Tukey $(\alpha=5 \%)$ evidenciou médias menores de densidade óptica no grupo 2 (glicocorticóide, 165,54 $\pm 8,95$ ), diferindo significativamente do grupo 1 (controle, 182,96 $\pm 4,53$ ) e do grupo 3 (risedronato, $180,99 \pm 6,21)$. Os grupos 1 e 3 não diferiram entre si. Os mesmos testes estatísticos evidenciaram diferenças significativas na proporção trabecular entre os três grupos. O grupo 2

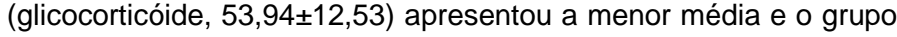
3 (risedronato, $81,14 \pm 7,20$ ), a maior. Ambos diferiram

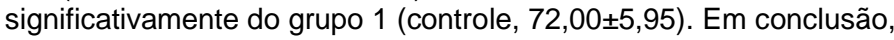
a administração de glicocorticóides e bifosfonatos altera a proporção de trabéculas ósseas na mandíbula de ratas e estas mudanças podem ser evidenciadas radiograficamente.

Palavras-Chave: Osteoporose; Rato; Mandíbula; Radiografia.

\author{
${ }^{1}$ Doutora em Clínica Odontológica; Professora Adjunta - Radiologia Odontológica \\ e Imaginologia, Universidade Luterana do Brasil \\ ${ }^{2}$ Estudante de Graduação, Universidade Luterana do Brasil \\ ${ }^{3}$ Estudante de Graduação, Universidade Luterana do Brasil \\ ${ }^{4}$ Bióloga, Laboratório de Patologia Bucal, Universidade Federal do Rio Grande do \\ Sul \\ ${ }^{5}$ Doutora em Estomatologia; Professora Adjunta - Radiologia Odontológica e \\ Imaginologia, Universidade Luterana do Brasil e Universidade Federal do Rio \\ Grande do Sul
}

Correspondência: Vania Fontanella

Endereço: Rua Cel Paulino Teixeira, 169/403 - CEP 90420-160 Porto Alegre RS, Brasil

E-mail: vaniafontanella@terra.com.br

Data de Submissão: 14/09/2008

Data de Aceite: 23/09/2009

\section{Introdução}

A possível relação entre perda óssea mandibular e a osteoporose foi inicialmente pesquisada devido à importância da integridade óssea para o sucesso de inúmeros tratamentos odontológicos (BIRKENFELD et al., 1999). Desde então, vários modelos de mensuração de massa e densidade óssea vêm sendo propostos, assim como meios de se avaliar a quantidade e a qualidade do tecido ósseo. O volume do osso trabecular na mandíbula é menor que o cortical, fator que pode dificultar a detecção de sua perda em indivíduos com osteoporose sistêmica (JIANG et al., 2004).

\section{Revisão da Literatura}

O aumento do risco à osteoporose e, conseqüentemente, na incidência de fraturas pode ser associado ao tratamento com glicocorticóides em altas doses e por prolongado tempo de administração (CANALIS; GIUSTINA, 2001; TAKAHASHI et al., 2004; BOLLING, 2004; COOPER, 2006; MAZZIOTTI et al., 2006; JUTEA et al., 2006). No tratamento da osteoporose induzida por glicocorticóides, o padrão ouro no tratamento farmacológico é o bifosfonato. Dos bifosfonatos, o risedronato sódico apresenta maior potência de ação, melhor tolerância em sua administração por via oral e menores efeitos colaterais (GARTON, 2001; PIAI et al., 2005).

Para avaliar a eficácia de agentes farmacológicos na prevenção e no tratamento da osteoporose, recomenda-se o uso de modelos animais, especialmente 0 de ovariectomizadas (THOMPSON, 1995; SHIRAISHI et al., 2009) ou medicadas com altas doses de glicocorticóides (TAKAHASHI et al., 2004; KOZAl et al., 2009).

A histomorfometria ou histologia quantitativa consiste na contagem ou medida dos componentes celulares e avaliação de alteração na microarquitetura óssea. É um método que permite a medida da mineralização e o estudo da formação óssea, 
especialmente na osteoporose induzida por glicocorticóides, caracterizada por adelgaçamento das trabéculas sem perfuração, que leva a uma redução no volume do osso (HUANG et al., 2004; ZECCHIN et al., 2004; CARBONARE et al., 2005; PIAI et al., 2005).

Por outro lado, os métodos radiográficos convencionais têm pouca precisão e baixa sensibilidade, só evidenciando a perda de massa óssea quando esta for superior a 30\% (YANG et al., 2005). Contudo, na radiografia digital a característica de subjetividade é eliminada, aumentando a precisão do diagnóstico, pois este método possibilita a identificação de sutis variações de densidade óptica (DO), as quais dificilmente seriam detectadas pelo olho humano (KHADEMI, 1996).

Desta forma, o objetivo do presente estudo foi verificar se a administração de glicocorticóide e bifosfonato alteram a proporção trabecular no osso mandibular e se estas alterações se expressam em termos de densidade óptica.

\section{Metodologia}

O presente estudo, caracterizado como ensaio clínico controlado e randomizado em modelo animal, foi aprovado pelo Comitê de Ética em Pesquisa da Faculdade de Odontologia da Universidade Federal do Rio Grande do Sul, protocolo $n^{0} \cdot 42 / 06$.

Foram utilizadas 36 fêmeas de Rattus Norvegicus da linhagem Wistar com 13 semanas de idade e peso entre 150 e 200 g, adquiridas do Biotério da Universidade Luterana do Brasil. As ratas foram identificadas por grupo com cores distintas na cauda e mantidas em gaiolas específicas, em grupos de cinco por gaiola, em ambiente com temperatura controlada de $23 \pm 3^{0} \mathrm{C}$ e umidade relativa do ar de $55 \pm 15 \%$, com ciclo claro e escuro de $12 \mathrm{~h}$. Foram alimentadas com ração padrão de laboratório e água ad libitum.

As ratas foram divididas em três grupos de 12 animais, que foram tratados por doze semanas, sendo o grupo 1 (controle) três vezes por semana, com injeções subcutâneas $(2 \mathrm{ml} / \mathrm{kg})$ de solução salina. O grupo 2 (glicocorticóide) recebeu acetato de metilprednisolona (1 mg/kg) em suspensão e diluído em solução salina. O grupo 3 (risedronato) recebeu o mesmo tratamento que o grupo 2 e foi tratado adicionalmente com risedronato $(3 \mathrm{mg} / \mathrm{kg})$ diluído em água destilada em volume de $5 \mathrm{ml} / \mathrm{kg}$ por doze semanas, seis vezes por semana, por via oral.

Os animais foram mortos, sob anestesia intraperitonial com Thiopental sódico (Thiopentax, $40 \mathrm{mg} / \mathrm{kg}$, Cristália, São Paulo, São Paulo, Brazil), após 12 semanas (grupos 1 e 2) e 24 semanas de tratamento (grupo 3). As hemi-mandíbulas esquerdas foram dissecadas e as peças anatômicas obtidas foram acondicionadas individualmente em potes plásticos identificados, contendo solução de formalina neutra tamponada $10 \%$.

As hemi-mandíbulas foram radiografadas com aparelho de raios $X$ Dabi Atlante (Ribeirão Preto, São Paulo, SP), com regime de $50 \mathrm{kV}, 8 \mathrm{~mA}$ e $0,1 \mathrm{~s}$, utilizando filmes periapicais no. 2 (Ektaspeed Plus, Kodak, Rochester, EUA). A peça foi colocada em uma placa de cera utilidade sobre uma base e mantida fixa sobre o filme. O cilindro localizador do aparelho foi adaptado a um suporte, de maneira a se obter angulagem vertical de $90^{\circ}$ e distância focal de $30 \mathrm{~cm}$. Os filmes foram processados pelo método temperaturatempo, simultaneamente, utilizando soluções novas (Kodak, Rochester, EUA)

As imagens foram digitalizadas em 300 dpi e salvas em formato BMP (Bitmap for Windows), importadas para o programa Adobe Photoshop CS (versão 7.0, Adobe Systems Inc., San Jose, CA, EUA). Sobre a imagem foi marcada, de forma padronizada, uma área de interesse para a análise, localizada na região do osso alveolar, abaixo das raízes do segundo molar (Figura 1). Desta região foram obtidos os valores médios e de desvio-padrão de DO, utilizando a função histograma do mesmo software, por um observador previamente treinado e cego para o grupo a que pertencia a imagem em avaliação.

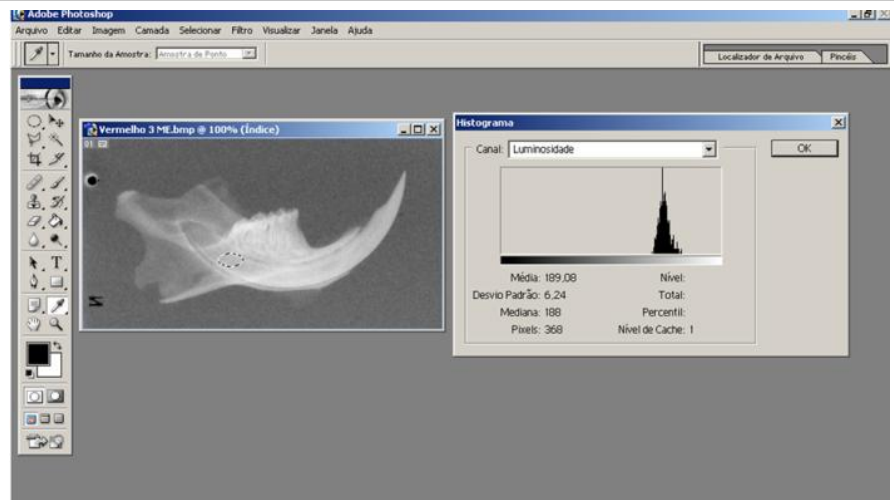

Figura 1. Obtenção dos valores de DO no programa Adobe Photoshop. A média e o desvio-padrão de DO da área demarcada na imagem são expressos na caixa de resultados do histograma.

As amostras foram descalcificadas em uma solução 1:1 de ácido fórmico $50 \%$ e citrato de sódio $20 \%$, no Laboratório de Patologia Bucal da FO-UFRGS. As mesmas foram mantidas imersas na solução, sendo a substância renovada diariamente, durante o período de descalcificação. O controle do tempo de descalcificação foi realizado mediante a tentativa de transfixação do espécime com uma agulha histológica.

Os espécimes descalcificados foram cortados com bisturi de forma a separar a região dos primeiros e segundos molares segundos molares e estas peças foram processadas para inclusão em parafina. Foram realizados três cortes semi-seriados longitudinais de $4 \mu \mathrm{m}$ de espessura, distantes entre si $1 \mathrm{~mm}$, a partir da extremidade da peça que representa o corte anteriormente realizado, valendo-se de micrótomo rotatório Leica 2155 . Os mesmos foram coletados em lâminas histológicas. Posteriormente, realizou-se a coloração dos cortes empregando-se Hematoxilina e Eosina (HE) para observação em microscopia de luz transmitida.

Em cada corte histológico foi selecionada a área central, a qual foi capturada utilizando-se um microscópio óptico com aumento de quatro vezes acoplado a uma câmara digital (Nikon Coolpyx, Ayuthaia, Thailândia), com resolução de 300 dpi. As imagens capturadas foram importadas para o software Adobe Photoshop e as trabéculas ósseas presentes em cada campo tiveram sua área quantificada (Figura 2), visando à determinação da proporção de área entre trabéculas ósseas e espaços medulares. Os espécimes foram avaliados em etapas, sendo no máximo dez imagens diárias e, após um intervalo de 7 dias, a segunda avaliação. 


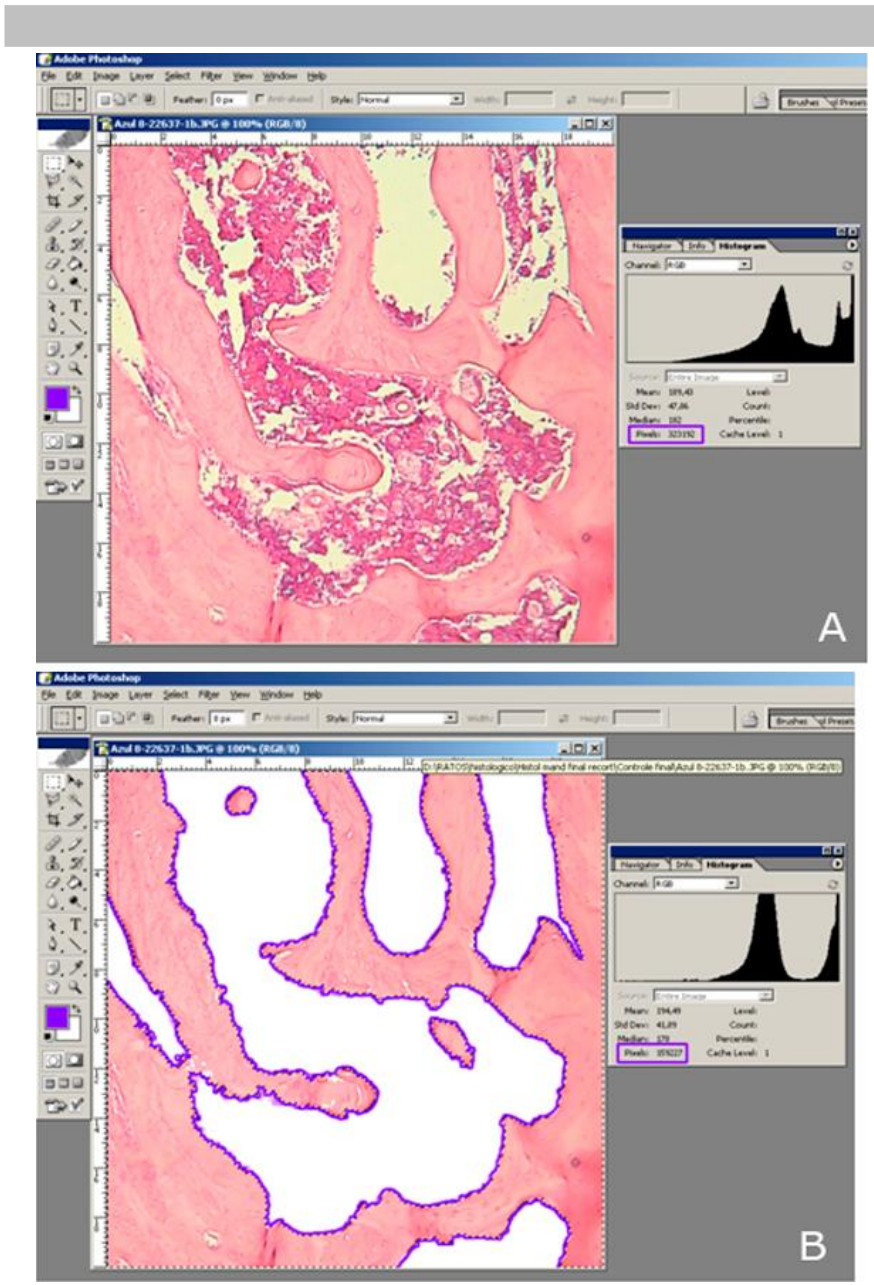

Figura 2. Obtenção, por meio da ferramenta histograma, da área total da imagem recortada $(A)$ e da área trabecular selecionada (B), expressas no histograma em pixels.

A Análise de Variância complementada pelo Teste de Comparações Múltiplas de Tukey, ao nível de significância de 5\%, foi empregada para verificação de diferença entre os grupos estudados, quanto aos valores de densidade óptica nas imagens e quanto à proporção entre trabéculas ósseas e espaços medulares. Para o processamento e análise destes dados foi utilizado o software estatístico SPSS versão 10.0.

\section{Resultados}

Por meio dos resultados da Análise de Variância verificase que existe diferença significativa entre os grupos comparados quanto à densidade óptica (Tabela 1). O teste de comparações múltiplas de Tukey indica que os grupos controle $(182,96 \pm 4,53)$ e risedronato $(180,99 \pm 6,21)$ apresentam as maiores médias (não diferindo entre si) e o grupo glicocorticóide $(165,54 \pm 8,95)$ apresenta a menor média, diferindo significativamente dos demais $(\mathrm{F}=23,55 ; \mathrm{p}<0,01)$.

Tabela 1. Comparação entre os grupos de estudo quanto à densidade óptica em radiografias da mandíbula.

\begin{tabular}{|c|c|c|c|c|c|c|}
\hline Grupo & $\mathrm{n}$ & Média* & Desvio-padrão & IC $95 \%{ }^{* *}$ & $\mathrm{~F}$ & $\mathrm{p}$ \\
\hline Cont & 12 & $182,96^{A}$ & 4,53 & {$[180,08$ a 185,84$]$} & 23,55 & $<0,01$ \\
\hline Glicocorticóide & 12 & $165,54^{\mathrm{B}}$ & 8,95 & {$[159,85$ a 171,22$]$} & & \\
\hline Risedronato & 12 & $180,99^{A}$ & 6,21 & {$[177,05$ a 184,93$]$} & & \\
\hline
\end{tabular}

* Médias 12 180,9 6,21

** Intervalo de confiança de $95 \%$ para a média
$\mathrm{Na}$ tabela 2, a ANOVA identifica diferenças estatísticas significativas entre os três grupos quanto à proporção trabecular. $\mathrm{O}$ Teste de comparações múltiplas de Tukey evidencia que o grupo 2 (glicocorticóide) apresenta a menor média de proporção de trabéculas ósseas por campo, seguido pelo grupo 1 (controle) e, por fim, com a maior média, encontra-se o grupo 3 (risedronato) $(F=37,56 ; p<0,01)$.

Tabela 2. Comparação entre os grupos de estudo quanto ao percentual de osso trabecular por campo.

\begin{tabular}{lrlllll}
\hline \multicolumn{1}{c}{ Grupo } & $\mathrm{n}$ & Média & Desvio-padrão & IC 95\%* & $\mathrm{F}$ & $\mathrm{p}$ \\
\hline Controle & 12 & $72,00^{\mathrm{A}}$ & 5,95 & {$[68,22$ a 75,78$]$} & 34,03 & $<0,01$ \\
Glicocorticóide & 12 & $53,94^{\mathrm{B}}$ & 12,53 & {$[45,98$ a 61,90] } & & \\
Risedronato & 12 & $84,14^{\mathrm{C}}$ & 7,20 & {$[79,56$ a 88,72] } & & \\
\hline
\end{tabular}

${ }^{*}$ Intervalo de confiança $95 \%$ para a média

** Médias seguidas de mesma letra não diferem entre si

\section{Discussão}

As imagens radiográficas digitalizadas deste estudo foram analisadas a partir dos níveis de cinza aferidos de uma determinada área, por meio da função histograma do programa Adobe Photoshop. A aplicação desta ferramenta é simples e reprodutível quando comparados resultados obtidos por diferentes observadores em tempos distintos (MAHL, 2003). Desta forma, justifica-se também o fato da análise ser realizada por um único observador.

Radiografias digitalizadas foram empregadas para calcular a densidade radiográfica em tons de cinza da área do osso alveolar. De acordo com Sarmento (2000), diferenças sutis na quantidade de tecido ósseo determinam significativas mudanças no nível de cinza de imagens digitalizadas.

Os resultados encontrados nesta pesquisa estão de acordo com Anbinder et al. (2007), que empregaram radiografias digitais de mandíbulas de ratas para calcular a DO do osso alveolar sob o primeiro molar inferior, em ratas com deficiência estrogênica e tratadas com bifosfonato e sinvastatina. No presente estudo, os valores de DO no grupo 2 (glicocorticóide) apresentaram-se estatisticamente diminuídos quando comparados àqueles do grupo 1 (controle), demonstrando que o tratamento induziu a perda de massa óssea. O grupo 3 (risedronato) apresentou aumento da DO, recuperando a massa óssea, pois resultou em valores semelhantes aos encontrados no grupo 1 (controle). Mahl e Fontanella (2008) demonstraram os mesmos resultados avaliando as radiografias por subtração digital.

Para analisar a proporção trabecular por campo foi utilizada a mesma área que que Iwasa et al. (2000) e Gorustovich et al. (2003). Observou-se que o corticóide induziu à diminuição do volume trabecular e que o risedronato recuperou a massa óssea, sugerindo que este método pode servir como modelo para estudo em distúrbios metabólicos ósseos.

\section{Conclusão}

A administração de glicocorticóides e bifosfonatos altera a proporção de trabéculas ósseas na mandíbula de ratas e estas mudanças podem ser evidenciadas radiograficamente.

\section{Referências}

ANBINDER, A. L. et al. The Influence of Ovariectomy, Simvastatin and Sodium Alendronate on Alveolar Bone in Rats. Braz. Oral Res., São Paulo, v. 21, no. 3, p. 247-252, July/Sept. 2007.

BIRKENFELD, L. et al. Menopause-related Oral Alveolar Bone Resorption: a Review of Relatively Unexplored Consequences of 
Estrogen Deficiency. Menopause, New York, v. 6, no. 2, p. 129 133, Summer 1999.

BOLING, E. P. Secondary Osteoporosis: Underlying Disease and the Risk for Glucocorticoid-induced Osteoporosis. Clin. Ther., Princeton, v. 26, no. 1, p.1-14, Jan. 2004.

CANALIS, E.; GIUSTINA, A. Glucocorticoid-Induced Osteoporosis: Summary of a Workshop. J. Clin. Endocrinol. Metab., Springfield, v. 86 , no. 12, p. 5681-5685, Dec. 2001.

CARBONARE, L. D. et al. Histomorphometric Analysis of Glucocorticoid-induced Osteoporosis. Mícron, Oxford, v. 36, no. 7 8, p. 645-652, Oct./Dec. 2005

COOPER, M. S. Sensitivity of Bone to Glucocorticoids. Clin. Sci. London, v. 107, no. 2, p. 111-123, Aug. 2006.

GARTON, M. J. Treatment of Osteoporosis with Bisphosphonates. CPD Rheumatology, [S.I.], v. 2, no. 3, p. 64-70, 2001.

GORUSTOVICH, A. A. et al. Mandibular Bone Remodeling Under a Choline-Deficient Diet: a Histomorphometric Study in Rats. J. Periodontol., Chicago, v. 74, no. 6, p. 831-837, June 2003.

HUANG, T-S. et al. Growth Hormone Cannot Enhance the Recovery of Dexamethasone-induced Osteopenia after Withdrawal in Young Female Wistar Rats. Tohoku J. Exp. Med., Tokyo, v. 204 no. 4, p. 257-266, Dec. 2004.

IWASA, Y. et al. The Effect of High Salt Intake on the Mandibular Bone Loss in Dahl-Iwai Salt-Sensitive Rat. J. Med. Dent. Sci. Tokyo, v. 47, no. 3, p. 187-195, Sept. 2000.

JIANG, G. et al. Prevention of Trabecular Bone Loss in the Mandible of Ovariectomized Rats. J. Oral Sci., Tokyo, v. 46, no. 2, p. 75-85, June 2004.

JUTEA, S. et al. Glucocorticoids Increase Inflamation-mediated Osteopenia in Rat. Acta Endocrinol., Bucharest, v. 2, no. 1, p. 1118, 2006.

KHADEMI, J. A. Digital Images \& Sounds. J. Dent. Educ. Washington, v. 60, no. 1, p. 41-46, Jan. 1996.

KOZAI, Y. et al. Influence of Prednisolone-induced Osteoporosis on Bone Mass and Bone Quality of the Mandible in Rats. Dentomaxillofac. Radiol., Houndsmills, v. 38 , no. 1, p. 34-41, Jan. 2009.

MAHL, C. E. W. Recursos Digitais no Diagnóstico Radiográfico de Reabsorção Radicular Externa Apical: Estudo In Vitro Simulando Movimentação Ortodôntica. Porto Alegre, 2003. 76 f. Dissertação (Mestrado) - Faculdade de Odontologia, Universidade Federal do Rio Grande do Sul, Porto Alegre.

MAHL, C. R. W.; FONTANELLA, V. Evaluation by Digital Subtraction Radiography of Induced Changes in the Bone Density of the Female Rat Mandible. Dentomaxillofac. Radiol. Houndsmills, v. 37, no. 8, p. 438-444, Dec. 2008.

MAZZIOTTI, G. et al. Glucocorticoid-induced Osteoporosis: an Update. Trends Endocrinol. Metab., New York, v. 17, no. 4, p. 144-149, May 2006.

PIAI, C. R. et al. Efeitos do Risedronato na Reparação Óssea de Ratos Machos e Fêmeas com Osteopenia. Cienc. Odontol. Bras. São José dos Campos, v. 8, n. 3, p. 77-82, jul./set. 2005.
SARMENTO, V. A. Diagnóstico Radiográfico de Alterações Periapicais de Origem Endodôntica através da Determinação do Nível de Cinza em Imagens Digitalizadas: estudo experimental em ratos. 2000. 284 f..Tese (Doutorado em Odontologia) - Faculdade de Odontologia, Pontifícia Universidade Católica do Rio Grande do Sul, Porto Alegre.

SHIRAISHI, A. et al. The Combination Therapy with Alfacalcidol and Risedronate Improves the Mechanical Property in Lumbar Spine by Affecting the Material Properties in an Ovariectomized Rat Model of Osteoporosis. BMC Musculoskelet. Disord., London, v. 10, no. 15 p. 10-66, June 2009 .

TAKAHASHI, K. et al. Effect of Incadronate on Corticosteroidinduced Osteopenia in Rats. Yakugaku Zasshi, Tokyo, v. 124, no. 9, p. 627-630, Sept. 2004

THOMPSON, D. D. et al. FDA Guidelines and Animal Models for Osteoporosis. Bone, Elmsford, v.17, no.4, p.125S-133S, Oct. 1995. YANG, J. et al. The Effect of Ovariectomy on Mandibular Cortical Thickness in the Rat. J. Dent., Kidlington, v. 33, no. 2, p. 123-129, Feb. 2005.

ZECCHIN, K. G. et al. Conventional X-ray Densitometry Detects Osteopenia in Ovariectomized Young Rats. Short communications. Braz. J. Oral Sci., Piracicaba, v. 3, no. 8, p. 425-427, Jan./Mar. 2004 